\title{
G. Schmidt. Thieme clinical companions: ultrasound, 5th edition.
}

\author{
Thieme Verlagsgruppe, Stuttgart, Germany, 2007. Softcover. 484 pp, 81 tables, 1091 \\ illustrations. Price: $€$ 39.95, CHF 67.90, \$ 49.95. ISBN 3-13-142711-6/978-3-13-42711-3
}

\author{
M. P. M. Stokkel • Luigi Mansi
}

Published online: 20 June 2007

(C) Springer-Verlag 2007

Advances in ultrasonography (US) over recent decades have resulted from improvements in equipment and new technologies as well as from the expansion of diagnostic capabilities. US contrast agents, colour Doppler sonography and improvements in guidelines have led to impressive progress in sonographic diagnosis.

This book presents a comprehensive overview of technical and clinical aspects of US. It is divided into three parts: the first part (grey part) is dedicated to basic principles and technical aspects, such as the physics of US, US examinations, artefacts, requirements for documentation, nomenclature and interventional US. This section is nicely illustrated with explanatory figures, anatomical illustrations and typical examples. In addition, not only are the capabilities of US described, but limitations and tips to increase the diagnostic yield are pointed out. The second part (green part) is dedicated to the investigation of principal signs and symptoms with US. Topics such as abdominal pain, unexplained fever, oedema, jaundice, goitre and joint pain and swelling are very well discussed and illustrated with typical sonographic findings. Each chapter describes the clinical manifestations, followed by diagnostic aspects, sonographic results and the accuracy of sonographic diagnosis. The third part (blue part) is dedicated to US of specific organs and organ systems, postoperative US and the search for occult tumours. Topics such as scan planes, sonographic anatomy and normal findings, scanning protocols, scanning tips and an explanation of findings are discussed. This part is richly illustrated with well-organised tables and typical sonographic images.

This book contains 21 chapters, 453 pages of text and a complete subject index. It is well written and illustrated with high-quality images and figures, and the standard format of each chapter means that it will be easily applicable in clinical practice. This book is more than a perfect guide to the use of US for medical students, residents and radiology technicians. It is highly recommended as a handbook in clinical practice for those who are performing US.
M. P. M. Stokkel

Leiden, The Netherlands

L. Mansi $(\bowtie)$

Naples, Italy

e-mail: luigi.mansi@unina2.it 